\title{
Naturgeschichte und Rassenklassifikation: Zu Blumenbachs Anthropologie und ihrer Rezeption
}

\author{
Per Oreste Sella, \\ maestro di familiare allegria e laboriosità, \\ con affettuosa vicinanza ai suoi cari
}

\begin{abstract}
The present chapter discusses the development of Johann Friedrich Blumenbach's (1752-1840) racial classification by analysing the theoretical, methodical, and conceptual shifts, and the ambivalences inherent to such an influential and controversial intellectual enterprise. Special attention is paid to Blumenbach's iconography, to his conceptualisation of natural history as a science of natural mutability, and to the importance he attributed to the relationship between indirect sources and direct observation of nature in anthropological research. The chapter focuses on Blumenbach's doctrine of degeneration and his alleged anticipation of Eugen Fischer's domestication theory, as well as on the image of the book of nature and the 'end of natural history' (Lepenies), on the transition from the idea of 'variety' to that of 'race', and on the visualisation of human varieties. Finally, the problematic role of the notion of beauty in both the construction and reception of Blumenbach's classification is highlighted.
\end{abstract}

Im Folgenden wird Blumenbachs Beitrag zur physischen und philosophischen Anthropologie durch eine kritische Darstellung der theoretischen, methodischen und begrifflichen Grundlagen seiner Rassenklassifikation und ihrer Veranschaulichung untersucht. ${ }^{1}$ Im ersten, einführenden Abschnitt werden einige $\mathrm{zu}$

1 Der vorliegende Beitrag fasst für das deutschsprachige Publikum einige in meiner italienischen Edition der Beyträge zur Naturgeschichte (CSN) dokumentierten Ergänzungen zu dem von mir herausgegebenen Olms-Reprint derselben Schrift (BN) zusammen. Neu sind gegenüber dem letzteren 1. der Kommentar zu Blumenbachs Ikonographie, 2. der kritische Bezug auf Wolf Lepenies' Studie zum Ende der Naturgeschichte, 3. die Stellungnahme zu Mühlmanns und Luigi Marinos Debatte über Blumenbach und die Domestikationstheorie, 4. die kritische Berücksichtigung der aus der Genderforschung und Ideengeschichte stammenden Studien zur Idee der caucasischen Rasse, 5. die Bezüge auf die norma verticalis und die sogenannten 'Musterköpfe' der fünf hauptsächlichen Menschenrassen. Die beiden letzten Punkte sind auch gegenüber der ita-

Ә OpenAccess. (c) 2021 Mario Marino, publiziert von De Gruyter. (cc) BY-NC-ND Dieses Werk ist lizenziert unter einer Creative Commons Namensnennung - Nicht kommerziell - Keine Bearbeitung 4.0 International Lizenz. https://doi.org/10.1515/9783110674194-006 
Blumenbachs Lebzeiten und in unserer Gegenwart entstandene, Blumenbachs intellektuelles Erbe repräsentierende Bilder kommentiert, wodurch von vornherein auf einige theoretische Ambivalenzen und textuelle Probleme aufmerksam gemacht wird, die Blumenbachs Lehre und Rezeption bedingten. In einem zweiten Abschnitt werden die theoretischen und methodischen Prämissen wie auch die stetige Evolution von Blumenbachs Rassenanthropologie durch Rückgriff auf die Lehre der natürlichen und menschlichen Veränderlichkeit, auf die Definition der menschlichen Natur und den originellen Gebrauch des Bildes vom Buch der Natur beschrieben. Ein dritter und letzter Abschnitt fördert die begrifflichen Schwierigkeiten und methodischen Probleme zutage, die die Systematik von Blumenbachs Anthropologie und Klassifikation im allgemeinen und seine Auffassung und Definition der sogenannten ,caucasischen Rasse insbesondere betreffen.

\section{Einleitende Bemerkungen zu Blumenbachs intellektuellem Erbe und Ikonographie}

Der Name von Blumenbach ist aus mindestens vier Gründen in die Geschichte der Rassen- und physischen Anthropologie fest eingeschrieben: 1. wegen seiner mehr als 200 Stücke umfassende Schädelsammlung, die gegen Ende seines Lebens zu den weltweit größten und vollständigsten zählte, 2. der in kritischer Auseinandersetzung mit Petrus Camper entstandenen norma verticalis, also: der Sicht auf den Schädel von oben, durch welche die Technik der kraniologischen Messungen erheblich erneuert wurde (GH 1795, § 60, 200 - 203), 3. der Einteilung der Menschheit in fünf hauptsächliche Rassen, die als erste moderne Rassenklassifikation gilt, 4. der Etablierung des heute noch - z. B. von den US Citizenship and Immigration Services und beim kontroversen racial profiling - verwendeten Wortes ,caucasisch“ im physisch-anthropologischen Fachvokabular (zuerst von Christoph Meiners in einem grob rassistischen Kontext benutzt, wurde es von Blumenbach übernommen und neu besetzt, als Meiners es explizit aufgab: Baum 2006, 73, 84-89, 59). Vor solchem Hintergrund ist es kein Zufall, dass Stephen J. Gould Blumenbach vor 25 Jahren als „the geometer of race“ (Gould 2002) bezeichnete.

Ein Blick in Blumenbachs Ikonographie bestätigt die enorme Bedeutung des kraniologischen Motivs in der öffentlichen Wahrnehmung seines wissenschaftlichen Erbes und seiner Persönlichkeit. Das gängigste Porträt stammt von Ludwig

lienischen Edition vollständig neu. Eine umfangreichere Fassung des Beitrages erscheint auf Englisch für die internationale Leserschaft in Marino 2020 [http://hdl.handle.net/10077/30261]. 
Emil Grimm, dem jüngsten und am wenigsten bekannten unter den Brüdern Grimm (Abb. 1). Es wurde 1823 anlässlich eines Tea Afternoon im Hause des Porträtierten und in Anwesenheit einiger Damen, darunter Blumenbachs Frau, skizziert. Blumenbach sitzt zu Hause, wo er die ganze Sammlung tatsächlich aufbewahrte, neben dem Schädel von „Richard Bruce King of Scotland“. Wie und warum dieser Schädel Eingang in seine Sammlung fand, erzählte Blumenbach selbst beim Nippen an einem Tässchen Tee. Im Volksaberglauben besaß dieser Schädel die Zauberkraft, Menschen in mutige und tüchtige Anführer zu verwandeln. Da sich George IV, König von Großbritannien, Sorgen um das Wiedererwachen des nationalen Stolzes in Schottland machte, ließ er sich den Schädel besorgen, wusste aber nach einer Weile nicht mehr richtig, was er überhaupt damit tun sollte - er spürte offenbar keine Steigerung seiner militärischen und politischen Tugenden -, und so entschloss er sich, ihn Blumenbach zu schenken. Die Anekdote attestiert Blumenbachs Ironie gegenüber dem eigenen Forschungsobjekt und dessen zeitgenössischer Romantisierung sowie gegenüber dem maßgeblich durch sein Werk erhöhten wissenschaftlichen Status der physischen Anthropologie.

Zwei Jahre danach findet eine feierliche Zeremonie zum 50. Jahrestag von Blumenbachs Dissertation De generis humani varietate nativa in Göttingen statt. Finanziert wird die Gedenkmedaille (Abb. 2) von rund 1500 Subskribenten, hauptsächlich Kollegen, ehemaligen Schülern und Korrespondenten aus ganz Europa und dem Rest der Welt: Der erste Professor für Anatomie und Psychologie an der Berliner Universität, Karl Asmund Rudolphi, koordiniert die Initiative. Die lateinische Schrift auf der Medaille lautet auf der Vorderseite „I. Fr. Blumenbach nato Gothae d. 11. Maii 1752 doct. creato Gottingae“ bzw. auf der Rückseite „d. 19. Sept. 1775 naturae interpreti ossa loqui iubenti physiosophili germanici“. Auf der einen Seite ist ein offizielles Brustbild von Blumenbach zu bewundern, ein elegantes Kleid und die Auszeichnung des Hannoveraner Ritterordens tragend, auf der anderen bilden die Schädel der caucasischen, mongolischen und äthiopischen Rasse ein Dreieck mit dem caucasischen Schädel im oberen Winkel.

Problematisch ist dabei nicht nur das Bild auf der Vorderseite, das Blumenbachs Lebenseinstellung und seinen Werten eklatant widerspricht (http://www. blumenbach-online.de/Einzelseiten/Medaille1825.php, Abruf: 26.08.2019), sondern auch und gravierender noch das der Rückseite, sofern es eine zweideutige, vereinfachende und verzerrende Interpretation von ambivalenten Textstellen aus seinen Schriften bedient. Blumenbach spricht nicht von nur drei, sondern stets von vier und - seit Anbeginn der 1780er Jahre - fünf Hauptrassen, zugleich definierte er die amerikanische und malayische Rasse als „Übergänge“ zwischen der caucasischen - als Stammrasse - auf der einen Seite und der äthiopischen und mongolischen auf der anderen (HN 1821, 70) (vgl. Abb. 8-12). In der dritten 
Auflage der Dissertation stößt man auf eine Abbildung, die einzig und alleine die drei letztgenannten Schädel umfasst, diese dient allerdings nicht der Veranschaulichung der Klassifikation der Menschenrassen (womit Blumenbach bei der eigenen Annahme von fünf Hauptrassen konsequent bleibt), sondern explizit (GH 1795, 204) der Exemplifizierung der Aussagekraft der norma verticalis (und tatsächlich werden alle drei Schädel von oben angezeigt, Abb. 3). Während in den Abbildungen aus der Dissertation von 1795 die Schädel nebeneinanderstehen, besteht die Rückseite der Medaille ausschließlich aus frontalen oder lateralen Ansichten, deren hierarchische Zusammensetzung den Vorrang der caucasischen Rasse nahelegt. Unter solchen Prämissen fällt es schwer zu behaupten, dass die Medaille eine getreue Veranschaulichung von Blumenbachs Persönlichkeit, Lehre und Methodik bietet: Wenn Blumenbach (wie auf der Medaille eindringlich behauptet wird) bei seiner Deutung der Natur die Knochen sprechen ließ, dann leiteten Rudolphi und seine Kollegen diese Rede verzerrt und vereinfachend weiter. Eine Erklärung dessen, wie und inwieweit Blumenbachs Schriften diese und ähnliche Interpretationen begünstigten, gehört zu den Zielen des vorliegenden Aufsatzes: Auf das Begriffspaar „Hauptrassen“ und „Übergänge“ wurde schon hingewiesen, später komme ich auf Blumenbachs Verständnis der caucasischen Rasse zurück.

Zwei Bilder verdienen noch analysiert zu werden. $\mathrm{Zu}$ der Homepage des in Göttingen angesiedelten großen Digitalisierungsprojekts der vollständigen Schriften und Sammlungen Blumenbachs sind zwei Banner elaboriert worden. Das ältere geht auf das Jahr 2010 zurück: Rechts ist Blumenbachs Gesicht nach dem üblichen Porträt von Grimm, links ist ein Schädel erkennbar, der aber inzwischen als Buchstütze dient (Abb. 4). Diese Komposition scheint eine doppelte Botschaft zu senden: Blumenbach wird allseits noch mit seinen kraniologischen Interessen assoziiert, diese Interessen betrachtet die gegenwärtige Forschung aber als wissenschaftlich überholt. Das Großprojekt hat also keineswegs das Anliegen, Blumenbachs physische Anthropologie zu verbreiten oder zu aktualisieren.

Das neuere Bild (Abb. 5, https://www.blumenbach-online.de/index.php, Abruf: 17.05.2020), das dasjenige von 2010 endgültig ablöst, entstand 2018 und zeichnet sich dadurch aus, dass kein ironischer und dialektischer Zugang zur kraniologischen Anthropologie versucht wird. Die neue Webseite hat ein Banner mit einer vorprogrammierten Bildsequenz: Wenn die Homepage abgerufen wird, ist die Medaille von 1825 stets das erste gezeigte Bild der Sequenz. Klickt man auf die einzelnen Bilder, werden die entsprechenden Digitalisate visualisiert. Die Botschaft scheint diesmal die zu sein, dass Blumenbachs Erbe durch die für das gesamte Projekt kennzeichnende technische Innovation der Digitalisierung in 'seinem' Göttingen akademisch wiedergewonnen, ja neu institutionalisiert und - 
durch das Web - von Göttingen aus in die ganze Welt projiziert wird. Beide Tatsachen, dass nämlich im Rahmen dieses Projektes so abweichende und sowohl aus der ikonographischen wie auch der wissenschaftsgeschichtlichen Tradition schöpfende Visualisierungen des Umganges mit Blumenbach entstanden sind und dass eine reduktive Interpretation der Klassifikation als repräsentativ für die ganze Anthropologie Blumenbachs stilisiert wird (vgl. dazu Junker 1998 und Gould 1998), zeigen ausgiebig, wie ambivalent und problematisch die Rezeption und Interpretation der Anthropologie Blumenbachs sein kann.

\section{Veränderlichkeit der Natur und die Anthropologie als Teil der Naturgeschichte}

Sollte man nun den Sinn von Blumenbachs Naturgeschichte in eine Formel zusammenfassen, dann vielleicht in folgende: eine empirisch angelegte Wissenschaft von der Veränderlichkeit der Natur. Für Blumenbach sind alle Naturreiche dem Wandel unterzogen und selbst die Verschiedenheit der Menschen bringt zunächst und vor allem solche Veränderlichkeit zum Ausdruck. Die menschliche Vielfalt ist dementsprechend als Naturphänomen zu betrachten und die Anthropologie als integraler Bestandteil der Naturgeschichte zu betreiben, auch wenn manche Verschiedenheiten keine direkte natürliche Ursache haben, sondern als Wirkungen von technisch-kulturellen Transformationen auszulegen sind, die die Natur durch menschliche Eingriffe durchmacht. Um die Hervorbringung von intraspezifischen Varietäten organischer Wesen umfassend $\mathrm{zu}$ bezeichnen, bediente sich Blumenbach des Wortes „Degeneration“ (vgl. z. B. BN 1790, 33-49), das damals in der Naturgeschichte in diesem Zusammenhang üblich und mit Verfallstheorien nicht unbedingt verbunden war.

Eine allgemeine Systematik der Degenerationsursachen - sei es im Sinne der kausalen Mechanik oder der eineindeutigen Kausalität - erscheint Blumenbach als unmöglich. Er nimmt aber an, dass erstens die bereits im Hinblick auf die Naturkatastrophen ausgeschlossenen monokausalen Erklärungen hier fehl am Platz sind: Es gäbe eine Vielzahl von hauptsächlichen Ursachen, deren Wirkung zweitens auf spezifische Forschungsgegenstände nachweisbar sei (BN 1790, 35; BN 1806, 29). Beim ersten Punkt folgt Blumenbach dem größten Naturforscher seiner Zeit, Buffon, der Klima, Nahrung und Lebensformen als degenerative Hauptfaktoren herausgearbeitet hatte. Hinsichtlich des zweiten erforscht er die Wirksamkeit der Hauptursachen am Fallbeispiel der Schweine, sofern allgemeiner Konsens über deren Abstammung herrscht und sie als allesfressende, auf der ganzen Erde verbreitete Haustiere, die den Menschen am ähnlichsten sind, das 
aufschlussreichste und ihm am meisten zugehörige Objekt für die Erforschung der Degeneration und deren Ursachen bilden (BN 1790, 37-39, 47-48, 54; BN 1806, 33-35, 38, 46-47).

Solche Assoziation von Schwein und Mensch veranlasste Eugen Fischers Schüler Wilhelm Emil Mühlmann dazu, Blumenbach als Vorgänger von Fischers Domestikationstheorie zu preisen (Mühlmann 1968, 59, 188-189). Dazu äußerte sich Luigi Marino in seinem unübertroffenen Standardwerk zur kultur- und wissenschaftsgeschichtlichen Bedeutung der Universität Göttingen um 1800 eher skeptisch: Einerseits reichten die Quellen für eine zufriedenstellende Lösung des Problems nicht, andererseits gelte Blumenbachs Erkenntnisinteresse vorrangig der Rassenklassifikation (Marino 1975, 120). In der Tat lagen weder Mühlmann noch Marino richtig, wie sich im Folgenden nachweisen lässt. Mühlmanns Interpretation beruht auf einer Rekonstruktion von Blumenbachs Denkweg, wonach Blumenbach die Rassenunterschiede zunächst (1779) im Sinne der herkömmlichen Umweltlehre (Klima, Nahrung usw.) auslegte, bevor er dann (erst ab 1789) die Verschiedenheit der menschlichen Rassen mit der Degeneration der Haustiere verknüpfte. Ein perfekter Parallelismus von menschlichen und haustierischen Rassenmerkmalen ist aber in keiner Schrift von Blumenbach nachzuweisen. Neben diesem ersten, bloß negativen und indirekten Argument gegen Mühlmanns Rekonstruktion sei nun ein direktes und positives gestellt: Die Beyträge zur Naturgeschichte belegen explizit, dass die Erforschung der Haustiere keineswegs diejenige der Degeneration ersetzen soll. Ganz im Gegenteil fungiert die erstere genau deshalb als Bestätigung der letzteren, weil die Degeneration von Haustieren den Beweis der Wirkung von den Hauptursachen der Degeneration erbringt.

Ein drittes und entscheidendes Argument ist, dass die Assimilierung des Menschen an Haustiere eingeschränkt wird, indem sie angesprochen wird. Wo Blumenbach in den Beyträgen den Menschen als das vollkommenste unter den Haustieren definiert, dort pointiert er auch, dass von 'Haustier' im eigentlichen Sinne des Wortes und im Wesentlichen nur beim Menschen gesprochen werden kann (BN 1790, 48). Denn im Gegensatz zu allen Tieren, die man domestizieren kann, und gegen die Voraussetzung jeder Domestikationstheorie entwickelt sich der Mensch von keinem wilden Naturzustande aus: Sein ursprünglicher Zustand ist kein anderer als ein kultureller. Indem damit behauptet wird, dass der ursprüngliche Zustand des Menschen kein anderer als ein kultureller und sozialer ist, steht Blumenbachs Bestimmung des Menschen als Haustier in einer Linie mit der strukturellen Anthropologie von Claude Lévi-Strauss, der in seiner berühmten Studie über die elementaren Strukturen der Verwandtschaft jene Definition explizit und zustimmend wieder aufnimmt (Lévi-Strauss 2002, 5-6). Zugleich ließe sie sich an die Philosophische Anthropologie Gehlens anschließen, für welchen der Mensch von Natur aus ein Kulturwesen ist (Gehlen 2004, 3; Gehlen 1983, 171, 
239-240; Gehlen lehnt übrigens explizit die Hypothese eines wilden menschlichen Naturzustandes ab: ebd. 130-133). Tatsächlich ist der Mensch auch bei Blumenbach aus natürlichen - bei ihm: physiologischen (BN 1790, 50 - 55, hier insbesondere 54-55) - Gründen das Wesen, das innerhalb des Tierreichs die größte Plastizität, Welt- und Differenzierungsoffenheit und die geringste Abhängigkeit von einer begrenzten Umwelt aufweist.

Um solche Differenzierungen, Variierungen objektiv $\mathrm{zu}$ erörtern und $\mathrm{zu}$ klassifizieren, erarbeitet Blumenbach eine Methodik, die aus drei Regeln besteht (BN 1790, 59-61; BN 1806, 51-54). Die erste definiert die Anthropologie als ein vergleichendes naturalistisches Fach: Die Menschenerkenntnis erfolgt aus dem Vergleich der menschlichen organischen Strukturen mit denjenigen anderer Lebensarten, da der Mensch ein lebendiger Organismus unter anderen ist. Damit folgt Blumenbach sehr konsequent der Linnäischen Revolution - also der radikalen Einordnung des Menschen in das Natursystem. Die zweite Regel präzisiert die erste, indem geraten wird, sich den unmittelbaren Vergleichen zwischen entgegengesetzten Extremen zu enthalten. Denn die natürlichen Unterschiede sind subtilste Nuancen, feinste Schattierungen von einem Grad zum anderen: Übersieht man bei den Vergleichen die unzähligen Mittelstufen, dann sind die daraus resultierenden Unterschiede zu groß, um als Elemente ein und derselben Menge betrachtet zu werden und überhaupt aussagekräftig zu sein. ${ }^{2}$ Den theoretischen Hintergrund dieser zweiten Regel bilden unverkennbare Reminiszenzen an Buffons nominalistischem und antimetaphysischem Paradigma - ein Modell, das Herder und Forster inzwischen gegen Kant revidiert und ausgebaut hatten, indem sie es für die ganze Natur gelten ließen. Buffon und Blumenbach sind gemeinsam (1) der Gebrauch des Terminus „Nüance“, (2) die Idee, dass aus vergleichender Perspektive die Menschheit wie jede andere Spezies eine stufenhafte Reihe interner Differenzierungen bildet und (3) die Idee, dass Trennungen innerhalb solcher intraspezifischen Differenzierungen in der Tat „keine andere[n] als sehr willkührliche Grenzen zwischen diesen Spielarten“(BN 1790, 81) sind womit im Grunde behauptet wird, dass die Klassifikation etwas wesentlich anderes als eine Art Spiegel der Natur sei. Die dritte und letzte Regel sieht die Einrichtung einer möglichst breiten und gezielten Sammlung von empirischen Befunden anthropologischen Charakters vor, denn unsere Erkenntnis der Naturgeschichte beruht auf ,Anschauungen“ - hier im kantischen Sinne von sinnlicher Erkenntnis. Die wissenschaftshistorische und epistemologische Relevanz des

2 Vgl. dazu noch BN 1806, 69, wo die Idee des Organismus als eines „naturliche[n] System[s]“ die Vorschrift begründet, dass die Vergleichung das Ganze und nicht vereinzelte Merkmale umfassen soll, bzw. BN 1806, 77, wo die Vorstellung der Schönheit als Monopol einer einzigen Rasse relativiert wird. 
Bildes, durch welches Blumenbach diese letzte Regel veranschaulicht, verdient, dass man dabei kurz verweilt.

\subsection{Das Buch der Natur und das Ende der Naturgeschichte}

In der zweiten Auflage des ersten Teils der Beyträge (1806) schildert Blumenbach die letzte Regel der anthropologischen Forschung durch eine Metapher, die er schon in der ersten Auflage der Beyträge (1790) bei der detaillierten, 1806 dann weggelassenen (vgl. BN 1790, 62-78; BN 1806, 55-66) Erwiderung auf Christoph Meiners Kritik an seiner vergleichenden Studie der menschlichen Schädel eingebracht hatte. Gegen die vermeintliche Verallgemeinerung eines einzigen physisch-anthropologischen Maßstabs hatte Meiners eine Aufwertung anderer, beispielsweise historisch-geographischer Kriterien verlangt. Blumenbach hat nichts dagegen, gibt aber zu bedenken, dass ihn die Kritik nicht trifft, denn er macht keinen einseitigen, überzogenen und unangemessenen Gebrauch der physischanthropologischen Methode (BN, 1790, 67-68). Im Allgemeinen stimmt Blumenbach mit dem Göttinger Kollegen hinsichtlich der Grenzen der anschaulichen Erkenntnis wie auch ihrer nötigen Ergänzung durch zuverlässige Erfahrungen aus zweiter Hand überein: Das sei die gewöhnliche Vorgehensweise der Naturgeschichte. Blumenbachs Pointe liegt dennoch darin, dass man in der Naturgeschichte nicht berechtigt ist, die direkte und anschauliche Erkenntnis - in diesem Falle: die direkte, vergleichende Beobachtung von Schädeln - nur deshalb aufzugeben, weil sie - wie jede empirische Erkenntnis - nie erschöpfend und vollständig sein kann. Mit Blumenbachs Worten: Es ist „die unabbitliche Verpflichtung des Naturforschers [...], dass er auch alles anwenden müße, um vor allen Dingen sich so viele eigene Erfahrung als möglich zu verschaffen“ (BN 1790, 69). Beiläufig sei hier bemerkt, dass die Vorstellung, wonach die Grenzen der möglichen unmittelbaren Erfahrung erweitert und dabei nicht überschritten werden sollen, gewisse Analogien mit Kants Epistemologie aufweist, wobei die Bemerkung, dass die indirekte Erfahrung erst dann hinzukommt, wenn die direkte nicht weiterhilft, an Herders und Forsters Aufwertung der Quellenkritik in der Anthropologie erinnert. Wie später im Hinblick auf die dritte Regel der anthropologischen Forschung so variiert auch in der Replik auf Meiners Blumenbach originell Galileis Bild vom Buch der Natur:

Alle die Nachrichten von noch so fähigen und glaubwürdigen Zeugen [1806: „Alle Nachrichten, die man darüber, wenn auch mit möglichst critischer Vorsicht ausandern schöpft“] sind im Grunde doch für den Wahrheitsuchenden Naturforscher nichts mehr und nichts weniger [1806: „nichts weiter“] als eine Art symbolischer Bücher, die er mit guten Gewissen 
nie anders als quatenus unterschreiben kan, in so fern sie nemlich mit dem geoffenbarten Wort im Buch der Natur [1806: „mit dem geoffenbarten Buch der Natur"] übereinstimmen, und um diess zu beurtheilen muss er sich in diesem Buch so viel Belesenheit und dadurch eben so viel Erfahrung als möglich, verschaffen (BN 1790 69-70; BN 1806, 53; zu den Varianten, siehe $\mathrm{BN}$, xcvii- $\mathrm{xcviii).}$

Irrtümlicherweise hat Bruce Baum die Stelle als Evidenz dafür ausgelegt, dass „in 1806, tacking stock of his own scientific efforts, Blumenbach reflected on the ,symbolic' character of any reading of nature“ (Baum 2006, 91). In dieselbe Richtung geht Niekerk in seinem neuesten, übrigens innovativen BlumenbachBeitrag (Niekerk 2019, 31). Dabei wurde Baum von Thomas Bendyshes falscher Übersetzung der Formel „symbolische Bücher“ durch „a kind of symbolic writing“ (AT, 298; zu Bendyshes Leben und Persönlichkeit sowie zum ideologischen, kulturellen und institutionellen Hintergrund seines übersetzerischen Unterfanges: BN, ix - xv, lxxi - lxxviii) irregeleitet. Der wahre Sinn der Textstelle, der in der Veranschaulichung des richtigen Verhältnisses zwischen indirekter und direkter Naturerkenntnis besteht, erschließt sich in der Tat erst dann, wenn die theologische Bedeutung der Formel 'symbolische Bücher’ vergegenwärtigt wird. Diese bereits in der kirchenväterlichen Tradition attestierte Redewendung bezeichnete in der protestantischen Theologie des späten 17. Jahrhunderts den Textkorpus, der von den verschiedenen christlichen Sekten hervorgebracht worden war und ausschließlich für die eigene Glaubensgemeinschaft galt: so beispielsweise die verschiedenen Katechismen der protestantischen Kirchen. Im Laufe des 18. Jahrhunderts stellten zunächst die Pietisten und danach - und verstärkt - die Neologen die solchen Texten in der protestantischen Theologie zugeschriebene Normativität in Frage (Beutel 2009, 113). Und wenn man die Bedeutung der Erfahrung und der Empfindung in der - in Göttingen gut vertretenen - Neologie mitbedenkt, dann kann es wirklich nicht überraschen, dass sich Blumenbach dieser Formel bediente, um die Übergeordnetheit der direkten Erfahrung zu unterstreichen. Insgesamt besagt diese Textstelle, dass der Wahrheitssuchende möglichst viele direkte, sinnliche Kenntnisse sammeln muss, um das Buch der Natur überhaupt lesen zu können, und nur die Kenntnisse, die direkt in diesem Buch stehen, gelten als absolut wahr. Alle Informationen, die man aus anderen Büchern, d.h. aus indirekten Quellen wie den Reiseberichten schöpft, können dagegen nur insofern herangezogen werden, als sie mit den Informationen übereinstimmen, die man direkt aus dem Buch der Natur, d. h. aus der Beobachtung der Natur bezieht. Der ultimative Referenztext in der Naturforschung - das heilige Buch der Naturforscher - ist damit die Natur selbst, d. h. die direkte, empirische Beobachtung. Die indirekten Quellen stehen zu den direkten Quellen im gleichen Verhältnis wie die Katechismen und Glaubensbekenntnisse der jeweiligen Kirchen zur Bibel. 
Nun scheint diese Textstelle extra dafür geschafft zu sein, die These von Wolf Lepenies zum sogenannten ,Ende der Naturgeschichte“ (Lepenies 1976), also dem in den letzten Jahrzehnten des 18. Jahrhunderts rapide erfolgten Übergang von einer Naturgeschichte als Naturbeschreibung zur Idee der Geschichtlichkeit, Zeitlichkeit und Veränderlichkeit der Natur, zu besiegeln. Diesen kulturellen und epistemologischen Wendepunkt deutet Lepenies nämlich als Übergang von der Natur als Textkorpus zur Natur als Experimentier- und Beobachtungsfeld. In seiner Studie kommen aber leider weder speziell die Beyträge noch Blumenbachs Schaffen im Allgemeinen zur Geltung: Blumenbach findet nur sehr spärliche Erwähnung, meist in Bezug auf Kant.

\section{Die Entwicklung von Blumenbachs Klassifikation: Tendenzen und Probleme}

Unter den bisher geschilderten theoretisch-methodologischen Voraussetzungen entwickelt und revidiert Blumenbach seine anthropologische Klassifikation über eine Zeitspanne von mehr als 50 Jahren. Die Entwicklungslinien, Wendepunkte und Widersprüche dieses Unterfangens lassen sich anhand der einschlägigen Textstellen in den verschiedenen Ausgaben von De Generis Humani Varietate Nativa, Handbuch der Naturgeschichte, Beyträge zur Naturgeschichte und Abbildungen naturhistorischer Gegenstände aufzeigen.

Eine erste Tendenz betrifft die Terminologie: Mitte der 1780er Jahre hatte die Verwendung des Wortes ,Rasse“ im Hinblick auf die menschliche Verschiedenheit eine heftige Kontroverse zwischen Kant, Herder und Forster ausgelöst. Während sich Kant zwecks der Etablierung dieses Wortes im geschichtsphilosophischen Diskurs um eine begrifflich strenge Definition desselben bemühte, lehnte Herder das Wort ,Rasse“ kategorisch als ein ,unedles‘ ab. Blumenbach, der das Wort ,Varietät‘ oder ,Spielart‘ seit Beginn seiner Forschungstätigkeit bevorzugte, ließ sich letztlich von Kants Definition überzeugen und fing ab Mitte der 1790er Jahre an, das Wort ,Rasse“ unter Verweis auf Kant explizit und systematisch zu adoptieren (HN 1797, 23). Diese verhängnisvolle Entscheidung hat höchst wahrscheinlich strategische, ja wissenschaftspolitische wie auch theoretische Gründe. Einerseits hatte Kant zu dem Zeitpunkt, in welchem seine Philosophie die verbreitetste und einflussreichste in Deutschland war, Blumenbachs Lehre von der Organisation der lebendigen Körper öffentlich favorisiert und damit einen wesentlichen Beitrag zur philosophischen Legitimierung von Blumenbachs empirisch angelegter Naturforschung geleistet. Andererseits beschränkte Kant den Wortgebrauch auf die unausbleibliche Vererbung physischer und nur physischer 
Eigenschaften, dadurch die Illusion hegend, die menschliche Freiheit und Vernunft vor naturalistischen und biologistischen Reduktionismen zu schützen. Blumenbach war - anders als Kant - kein Dualist, wohl aber ein Humanist: Seine Anthropologie mobilisiert er voll und ganz gegen Sklaverei und Ausgrenzung von sozialen Gruppen sowie gegen die Abwertung von ganzen Bevölkerungen (BN 84-118).

Man kann allerdings noch einen weiteren, allgemeineren, mehr theoretischen Grund für die Ablösung von ,Varietät‘ und ,Spielart‘ durch ,Rasse‘ vermuten. Formeln wie ,Spielart‘ und Varietät‘ erschienen möglicherweise zu wenig spezifisch, indem sie den Akzent auf die aleatorische, ungewisse, äußere, willkürliche Seite der beobachteten Phänomene setzen: als ob die Natur mit den eigenen Erscheinungen spiele, ja selbst als Künstlerin aufzufassen sei. Solche Formeln konnten also suggerieren, dass der Forschungsgegenstand unwesentlich, nebensächlich und einer rationalen Erklärung teilweise oder unvollständig unzugänglich sei. Durch die Annahme eines zusammenhängenden und konstanten Sets von physischen Eigenschaften hatte Kants Bestimmung des Rassenbegriffs zur Verwissenschaftlichung, ja zur Verlagerung der Diskussion über die Natur und deren Vielfalt in die naturwissenschaftlichen Bereiche sowie zur Herstellung eines entsprechenden kategorialen Apparats beigetragen. Mit seiner Definition hatte Kant also - in Bezug auf die Sprachlichkeit und Begrifflichkeit der Anthropologie - die Weichen gestellt und Blumenbach musste früher oder später den einen oder den anderen Weg einschlagen. Blumenbachs Arbeit ging offensichtlich in die Richtung einer Grundlegung der Anthropologie als empirischer Wissenschaft der Natur, sodass die Übernahme des Kantischen Rassenbegriffs nachvollziehbar ist.

Eine zweite Tendenz ist der Übergang von einer bloßen Aufzählung zur Bezeichnung der jeweiligen Rassen und damit von einer ungeordneten zu einer systematischen Präsentation der anatomischen Daten (in der Reihenfolge: Hautfarbe, Haarfarbe und Wuchs, Gesichtszüge und Schädel). Eine dritte, parallel laufende und ebenso konstante Tendenz ist die Auslassung der nicht anatomischen und nicht geographischen, also: nicht physischen, zumal der bloß kulturellen und theologischen Daten (wie z.B. die im Handbuch ab 1788 bzw. 1791 fortfallenden Hinweise auf die Sprache der Finnen und auf Adam als Stammvater des Menschengeschlechts, HN 1788, 60-61; HN 1791, 54-55). Innerhalb der Klassifikation gibt es eine einzige explizit von kulturellen Werten abhängige Betrachtung, die in allen Ausgaben des Handbuchs erhalten bleibt: Die caucasische Rasse, repräsentiert vom Schädel einer Georgierin, habe die „nach den Europäischen Begriffen von Schönheit musterhafteste [...] Schädel- und Gesichts-Form“ (HN 1797, 61; zu vergleichen mit HN 1830, 56; in früheren Ausgaben des Handbuchs 
wird allgemeiner behauptet, die „Völker“ dieser Rasse seien nach den eben genannten Maßstäben die „bestgebildetsten Menschen“, z. B. in HN 1788, 61).

Wieso hat Blumenbach darauf immer bestanden? Angenommen, dass die wissenschaftliche Legitimierung und Geltung der Klassifikation auf eine möglichst objektive und physisch orientierte Methodologie angewiesen war, dann würde jene Aussage die ganze theoretische Grundlegung der Klassifikation abschwächen. Meines Erachtens liegt der Grund für diese Insistenz darin, dass er den caucasischen Schädel tatsächlich als den physisch wohlproportioniertesten betrachtete. Das heißt: Jenes kulturelle Urteil über die caucasische Schönheit hatte bei Blumenbach eine objektive, physische, geometrische Referenz - die übrigens begrifflich und systematisch von entscheidender Bedeutung für seine Anthropologie ist. Vor dem Hintergrund der Klima- und Temperamenttheorie einerseits und anhand von physiologischen Betrachtungen andererseits tendiert Blumenbach nämlich dazu zu glauben, dass (1) die in temperierten Klimazonen lebenden Bevölkerungen eine proportioniertere Verfassung bilden, (2) der Übergang von weißer zu schwarzer Hautfarbe leichter erfolgt als umgekehrt und (3) die temperierten Klimazonen dem Menschen am Anfang seiner Naturgeschichte günstigere Lebensbedingungen bieten. Die kraniologischen Untersuchungen unterstützen diese Tendenz, indem die Anwendung der norma verticalis drei ideelle Schädelformen erkennen lässt, die ebenso vielen hauptsächlichen Menschengruppen entsprechen: eine extrem symmetrische und beinah runde oder sphärische Form für die caucasische, eine sich in die Breite entwickelnde, abgeflachte und gewissermaßen quadratische für die mongolische und eine, die sich in die Länge entwickelt, für die äthiopische.

Auf der einen Seite zeigt sich Blumenbach dessen bewusst, dass die Gleichsetzung von harmonischen Verhältnissen und Schönheit eine kulturelle Gegebenheit der europäischen, zumal altgriechischen Tradition ist. Auf der anderen scheint er sich aber der kulturellen und theoretischen Bedingtheit seiner eigenen Annahmen nicht völlig bewusst zu sein, wonach der georgische Schädel als Muster des caucasischen im Allgemeinen anzusehen ist, also die vermeintliche Objektivität und Vollkommenheit seiner Proportionen die Klassifikation der caucasischen als „Stamm-“ und „Mittel-Rasse“ mit ermöglicht.

Wohl gemerkt: In Blumenbachs Anthropologie handelt es sich bei diesem Begriffspaar keineswegs um einen Ausgangs-, sondern eher um einen Endpunkt. Als er die Rasse, die später caucasisch genannt wird, in der ersten Ausgabe des Handbuchs einführt, beschreibt er sie als die „größte“ und „ursprüngliche“ (HN 1779, 63), ohne dabei das genealogische Verhältnis der übrigen klassifizierten Menschengruppen zu dieser ersten auch nur anzudeuten. Als in der vierten Auflage des Handbuchs der Stammvater der Menschheit nicht mehr Adam ist, sondern viel prosaischer eine gemeinsame Stammrasse, wird diese mit keiner 
der fünf klassifizierten Rassen (nicht einmal mit der später in ,caucasisch umgenannten) identifiziert; die Bezeichnungen „größte“ und „ursprünglich“ fallen dementsprechend weg (vgl. HN 1788, 60; 1791, 54). Es geschieht erst nach der dritten Auflage der Dissertation (1795), dass die erste, noch vorläufige Bestimmung der caucasischen als der „mittlere[n] oder Stamm-Rasse“ in das Handbuch aufgenommen wird (HN 1797, 63). Ab 1799 stabilisiert sich dann die Definition, obwohl die dabei getroffene Formulierung etwas ungewiss und zweideutig bleibt („die sogenannte“, „oder“): „Von diesen fünf Haupt-Rassen muß nach allen physiologischen Gründen die caucasische als die sogenannte Stamm- oder MittelRasse angenommen werden“ (HN 1799, 64).

$\mathrm{Zu}$ bedenken ist nun, dass die so bezeichnete caucasische Rasse durch den Begriff der Stammrasse genealogisch, zeitlich-historisch und durch denjenigen der Mittelrasse morphologisch-komparativ, typologisch erfasst wird. Blumenbach hielt wahrscheinlich beide Herangehensweisen für konvergent und darum auch äquivalent. In dem von seiner im Denken des 18. Jahrhunderts wurzelnden Idee der Natur vorgegebenen theoretischen Horizont kann eine solche Äquivalenz allerdings nicht bestehen, ohne Widersprüche zu erzeugen oder den allgemeinen theoretischen Rahmen infrage zu stellen: Wenn nämlich die Natur ein Kontinuum von Nuancen ist, dem gegenüber sich jede Trennung als willkürlich erweist, dann ist jede Klassifikation - insbesondere diejenige der plastischsten unter den Tierarten - nicht als Naturspiegel zu betrachten, sondern lediglich als eine auf der Wahrnehmung und Typisierung von besonders signifikanten Vorkommnissen beruhende Modellierung. Genau das meint Blumenbach mit der eigenen Klassifikation im Kontext seiner eigenen Naturgeschichte. Ist die eine Gruppe in der Klassifikation aber nicht nur ein mittleres Modell zwischen zwei Endpolen, sondern - aufgrund solcher mittleren Position - der konkrete, historische und natürliche Ausgangspunkt des ebenso konkreten, historischen und natürlichen Differenzierungsprozesses der menschlichen Spezies, dann entsteht die Frage, ob das geschichtliche Kontinuum diskret geworden ist bzw. ob die Klassifikation bloß typologisch ist - kurzum, ob und inwieweit die Beziehung zwischen Typologie und Geneaologie theoretisch und methodologisch noch auf soliden Grundlagen ruht. Diese Frage soll nicht nur auf eine theoretische und methodologische Schwierigkeit hinweisen, sondern auch auf eine historische Transition zwischen zwei Epochen und zwei verschiedenen Weisen, Naturgeschichte zu verstehen und $\mathrm{zu}$ betreiben. 


\subsection{Schädel, Umwelten, Muster: Wie bildet man die menschliche Vielfalt ab?}

Die im ersten Teil kritisch reflektierte Ikonographie hat gezeigt, dass die Bezüge auf die kraniologische Forschung in der damaligen und heutigen Wahrnehmung von Blumenbachs Persönlichkeit und wissenschaftlichem Erbe unübergehbar und vorherrschend sind. Abschließend sei nochmals auf einige Bilder rekurriert, diesmal aber anthropologische Forschungsobjekte betreffend und Blumenbachs Schriften entnommen. Die Tafel Nr. 2 in der dritten Ausgabe der Dissertation ist wahrscheinlich die bekannteste und am häufigsten reproduzierte unter diesen Abbildungen: Es ist eine streng typologische und bloß physische. Wir sehen ganz deutlich die in den Proportionen des Schädels begründete zentrale, mittlere Position der caucasischen Rasse. Diese Tafel steht gemeinhin für die ganze Klassifikation und Anthropologie Blumenbachs, in der Tat zielt sie aber nicht auf die Abbildung der fünf menschlichen Varietäten an sich hin, sondern auf diejenige der fünf Varietäten von Schädeln, sofern diese die aufschlussreichsten Forschungsgegenstände in der Untersuchung der menschlichen Verschiedenheit sind (GH 1795, 198).

Es gibt aber ein umfassenderes und weniger bekanntes Bild, in welchem die kraniologischen Daten zwar berücksichtigt und eingebunden, aber nebensächlich werden: Das sind die 5 Vignetten, die im ersten Teil der Beyträge zur Naturgeschichte die fünf Varietäten des Menschengeschlechts repräsentieren (Abb. $7 \mathrm{a}-\mathrm{e}$ ). ${ }^{3}$ In der Tafel Nr. 2 der Dissertation hatte man es mit einem einzigen physischen, osteologischen Element zu tun, das von seinem anatomischen Kontext gänzlich getrennt wurde. In den Vignetten erscheint der Repräsentant der jeweiligen Rasse in seiner physischen Integrität und eingebettet in seiner sozialen Dimension - im Wesentlichen bestimmt von dem natürlichen und kulturellen Kontext. Vor dem Hintergrund des heute vorherrschenden kulturellen Paradigmas der Naturwissenschaften wäre man versucht, allein der Tafel eine wissenschaftliche Bedeutung beizumessen und die Vignetten als bloß dekorative Abbildungen abzutun. Das wäre aber eine völlig falsche Annahme. Wer bestellte die Vignetten und mit welchen Absichten?

Blumenbachs Briefwechsel mit dem Verleger der Beyträge, Dieterich, und dem Stecher Daniel Nikolaus Chodowiecki belegt, dass es Blumenbach war, der die Vignetten als wesentlichen Teil seines anthropologischen Projektes auffasste,

3 Weder Schiebinger (1993) noch Bindman (2002), die meines Wissens die einzigen Forscher sind, die diese Bildreihe wahrgenommen und kommentiert haben, konnten eine korrekte Interpretation der Vignetten liefern. 
sie bis in die feinsten Details bestimmte und von keinem anderen Künstler als Chodowiecki stechen lassen wollte. In zwei Briefen vom 17. Dezember 1781 instruiert er Chodowiecki durch präzise Verweise auf die eigene Dissertation und ausgewählte Reiseliteratur und macht Dieterich klar, die Vignetten seien „nicht etwa blos zur Zierde des Buchs, sondern wesentlich nothwendig“, und könnten weder von Johann August Rosmäßler noch von Georg Gustav Endtner, nicht einmal von Johann Wilhelm Meil, sondern „blos von Chodow[iecky] recht gemacht werden“ (BC 1, Nr. 177, 293; zu diesen Künstlern und Chodowiecki: Marino 2020, 64-65: http://hdl.handle.net/10077/30261). Zwar war Codowiecki damals der am meisten gesuchte, gerühmte, kostspielige und erfolgversprechende Stecher Deutschlands (Selwyn 1997, 15), der Grund, weshalb Blumenbach ihn und keinen anderen für seine Beyträge wollte, ist allerdings nicht kommerzieller Art. Blumenbachs Zeitgenossen und die heutigen Kunsthistoriker sind sich darin einig, dass Chodowiecki für eine Kunst stand, die dem Prinzip der Naturnachahmung verschrieben war und dass er deshalb bewundert wurde, weil er den sozialen und natürlichen Kontext der abgebildeten Handlung mit der individuellen leiblichen Expressivität der handelnden Figuren unübertroffen in Verbindung zu setzen wusste. Und das war es genau, was sich Blumenbach ästhetisch und intellektuell für seine Schrift wünschte. Denn die Vignetten der Beyträge veranschaulichen offensichtlich die Lehre der menschlichen Varietäten als Produkte der Wechselwirkung von natürlichen und kulturellen Faktoren: die natürlichen Eigentümlichkeiten, die die physische Anthropologie und die Geographie feststellen - Hautfarbe, somatische Züge, Habitat - und die kulturellen Eigentümlichkeiten, die die damals beginnende Ethnologie erforschte und die mit den Lebensformen, ja letztendlich den ökonomischen Produktionsweisen zusammenhängen.

Es stellt sich indes die Frage, in welchem Verhältnis die Tafel aus der Dissertation und die Vignetten aus den Beyträgen zueinander stehen. Zwei Antworte sind möglich: Entweder ersetzt die spätere Tafel die früheren Vignetten oder beide ergänzen sich. Gegen die erstere spricht aus philologischer Sicht, dass Blumenbach in der zweiten Auflage (1806) des ersten Teils der Beyträge mehrere naturgeschichtlich relevante Textstellen und Kapitel (beispielsweise über den Ursprung des Basalts, die Teleologie, die Methodik und die Klassifikation selbst) aktualisierte und auch drastisch modifizierte, die Vignetten von Chodowiecki aber nicht entfernt wurden. Für die letztere spricht noch aus theoretischer Sicht, dass die Tafel und die Vignetten eindeutig für zwei verschiedene Herangehensweisen stehen: Die eine basiert auf der Degenerationslehre, die andere auf der kraniologischen Untersuchung. Wichtig ist nun, dass solche Ansätze in Blumenbachs Schriften koexistieren, sodass anzunehmen ist, dass es sich bei den Vignetten und 
den Tafeln um zwei methodisch verschiedene und doch komplementäre Sichtweisen auf ein und dasselbe Objekt handelt.

Diese These wird verstärkt durch die in der Schrift Abbildungen naturhistorischer Gegenstände veröffentlichte Sequenz der sogenannten fünf charakteristischen Musterköpfe der fünf Hauptrassen. Die Abbildungen sind unmissverständlich eine Publikation, die wissenschaftlichen Charakter aufweist und wissenschaftliche Ziele verfolgt. Abgesehen von den Textverweisen in Blumenbachs akademischem Text par excellence, dem Handbuch der Naturgeschichte, wo die Abbildungen als wissenschaftlicher Quellenapparat angeführt werden (HN 1807, xii, 67-69, 73-74), gibt es noch direktere, aussagekräftigere Beweise im Vorwort zu den Abbildungen. Hier werden die Abbildungen als ein Repertoire von epistemischen Objekten aufgefasst, das in eindeutiger Kontinuität mit der Doktrin des Verhältnisses von direkten und indirekten Kenntnissen eingerichtet wurde, die Blumenbach anhand der Metapher des Buches der Natur erläutert hatte (AG: Vorbericht, ohne Seitenzahlen). Laut Vorwort sollen die Abbildungen sowohl als komplementärer Text zum Handbuch als auch im Allgemeinen als Hilfsmittel in der Naturforschung dienen. Noch interessanter ist, dass insbesondere die Musterköpfe in suggestiv verdichteter Form wesentliche Elemente von Blumenbachs Anthropologie verkörpern. Solche sind: (1) die physischen Grundlagen der Klassifikation (Form des Gesichts und des Schädels), (2) die kulturellen Faktoren der menschlichen Veränderlichkeit, also der sogenannten Degeneration (die Art und Weise, wie Haar und ggf. Bart getragen werden sowie die Kopfbedeckung und die sonstige Bekleidung gelten als kulturelle Merkmale der jeweiligen Bevölkerung mit der einzigen Ausnahme des sogenannten ,Negers'), (3) die humanistischen Grundsätze und Grundwerte der Anthropologie Blumenbachs. Als Repräsentanten der fünf Hauptrassen gelten vorbildlich tugendhafte und talentierte Menschen der Kunst (Maler), der Sitten (Gentleman), der Wissenschaft (Theologen) und der Politik (Diplomaten und Anführer). Außerdem behauptet Blumenbach explizit, dass diese fünf Porträts, aus der Nähe betrachtet, den kraniologischen Modellen in den Tafeln der Dissertation von 1795 völlig entsprechen (AG: Characteristische Musterköpfe von Männern aus den 5 Hauptrassen im Menschengeschlecht, ohne Seitenzahlen). Bemerkenswert ist noch, dass die Abbildungen - anders als bei den Vignetten der Beyträge - keine entsprechenden natürlichen Umwelten reproduzieren: Dies bedeutet keine Revision der Degenerationslehre im Sinne einer Ausschließung der natürlichen Umwelt als Mitursache; die daraus entstehende Hervorhebung der physischen und der kulturellen Gegebenheit kann aber prinzipiell zu einschränkenden Deutungen der Klassifikation verleiten.

Zusammenfassend gesprochen scheint mir, dass Blumenbach nicht alle Ambivalenzen seiner Doktrin zu lösen vermochte. Dazu gehören die Miteinbeziehung der Schönheit als ein bestimmendes Merkmal der caucasischen Rasse 
und die unscharfe Unterscheidung zwischen Geschichte als Beschreibung und Geschichte als Genealogie bzw. nominaler und genealogischer Klassifikation. Der Rassismus des 19. Jahrhunderts löste solche Widersprüche, indem er einige doktrinäre Aspekte einseitig entwickelte. So begünstigen Blumenbachs theoretische Mängel und Ambivalenzen bei der Bestimmung des Status und der Eigenschaften der sogenannten caucasischen Rasse eine radikal rassistische Auslegung seiner ganzen Rassenklassifikation und Anthropologie. Das bezeugt paradigmatisch ein klassisches Werk der britischen Anthropologie wie die Universitätsvorlesungen zur Naturgeschichte von William Lawrence, selber ein Übersetzer und Bewunderer Blumenbachs (Lawrence 1822, 290 - 292). Einerseits wurde die Vorstellung der caucasischen Schönheit gänzlich naturalisiert, andererseits wurden moralische und geistige Fähigkeiten durch analogisches Verfahren kategorisch abgeleitet. Die physische Schönheit verwandelt sich nun in eine vermeintlich objektive: eben physische - unbestreitbare Grundlegung einer absoluten moralischen und geistigen Überlegenheit. Begründet wird dadurch eine rassische Hierarchie, gerechtfertigt eine imperiale und koloniale Herrschaft und Ausnutzung. Alle Rassen können nun von der caucasischen nach einem Verfallsprinzip abgeleitet werden. 


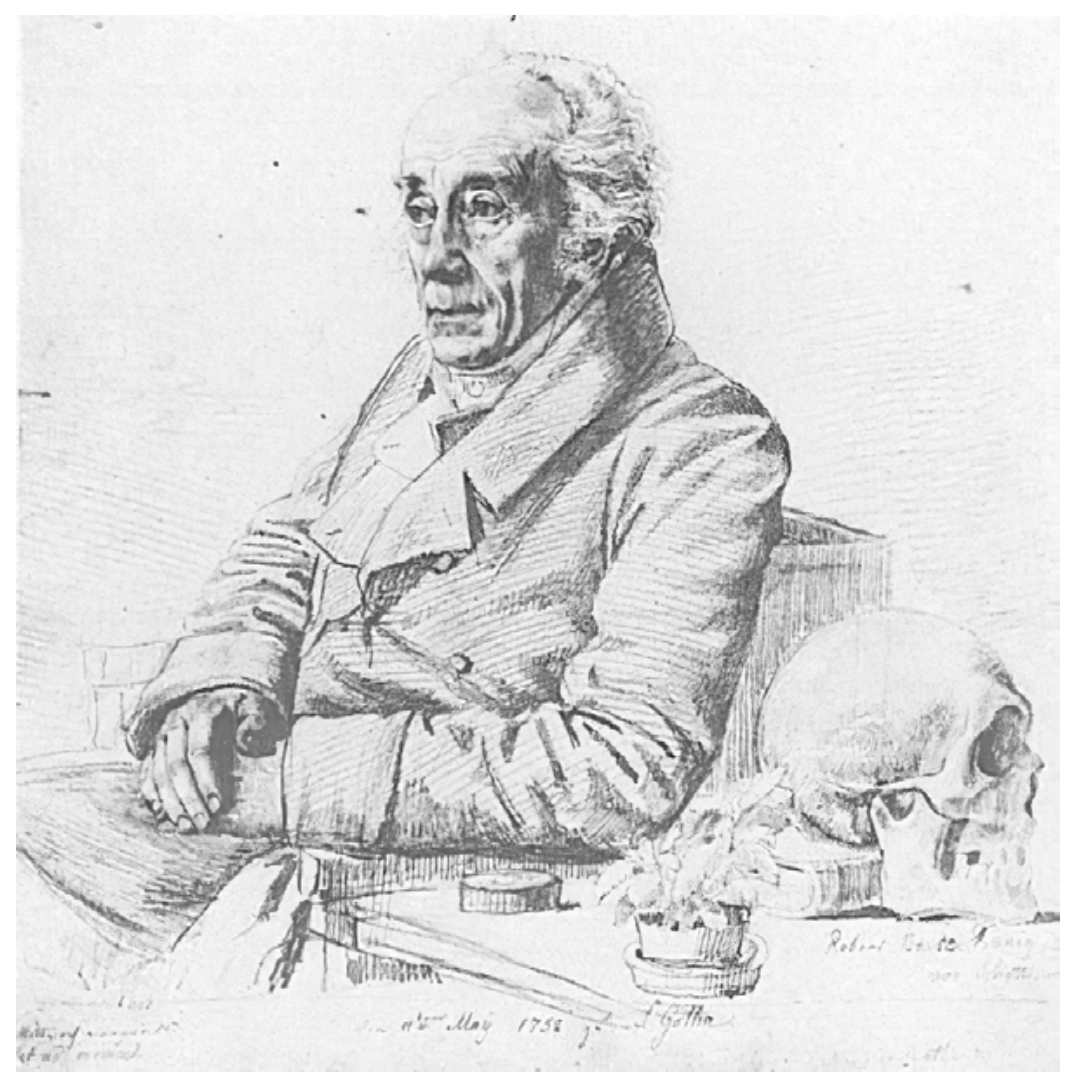

Abb. 1: Johann Friedrich Blumenbach (L. E. Grimm 1823).
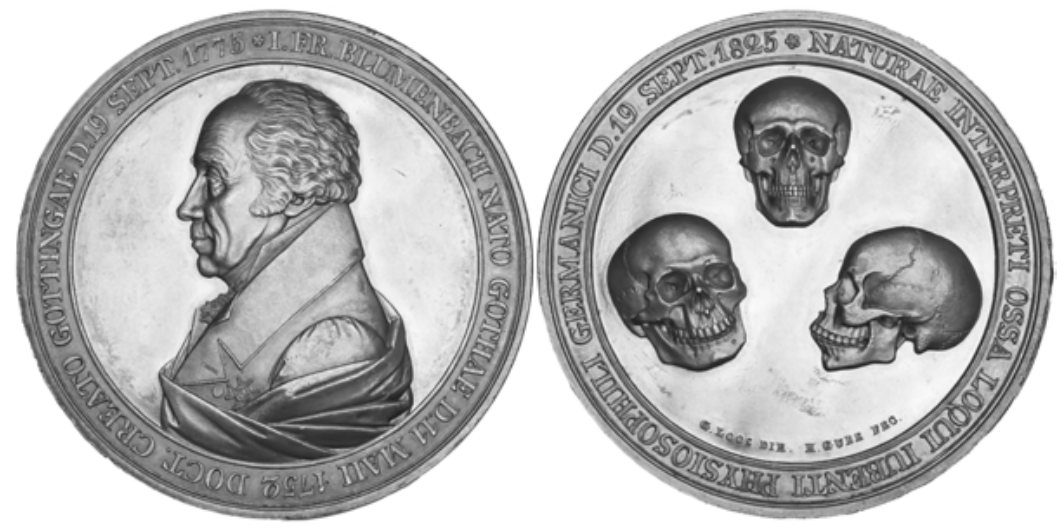

Abb. 2: Gedenkmedaille 1825. 

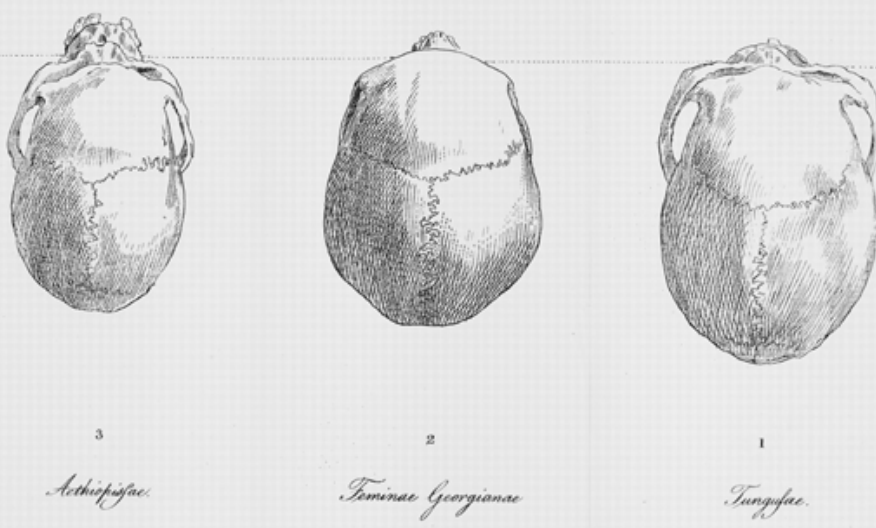

1

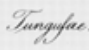

Abb. 3: J.F. Blumenbach: De generis humani varietate nativa (1795): Tab. I.

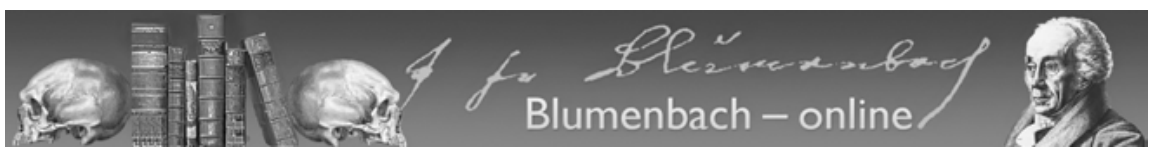

Abb. 4: www.blumenbach-online.de: Banner 2010.

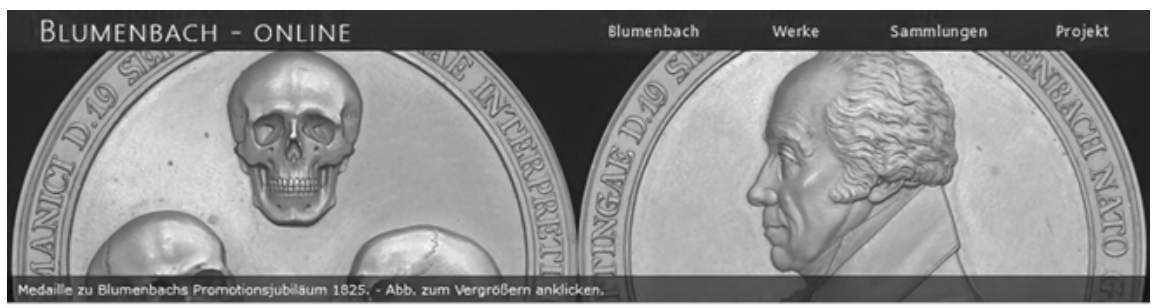

Johann Friedrich Blumenbach - online

Das Projeit Johann Friedrich Blumenbach - online- bearbeitet die Publikationen und die naturhistorischen Sammlungen des Gottinger Naturforschers Johann Friedrich Elumenbach (1752-1840). Ziel ist eine digitale Ausgabe seiner Werke und der erhaltenen Sammlungsobjekte.

Johann Friedrich Blumenbach - online- ist ein Langzeitprojekt im Rahmen des Akademienprogramms der Union der deutschen Alademien der Wissenschaften, angesiedelt bei der Akademie der Wissenschaften zu Gottingen.

Die Internetseite www.blumenbach-online.de

informiert uber L. F. Blumenbach und uber online-Ressourcen for die Blumenbach-Forschung, stell die bisher erarbeiteten Materialien in einer vorlaufigen Form zur Verfogung und prasentiert das Projelt.

Abb. 5: www.blumenbach-online.de: Banner 2018. 


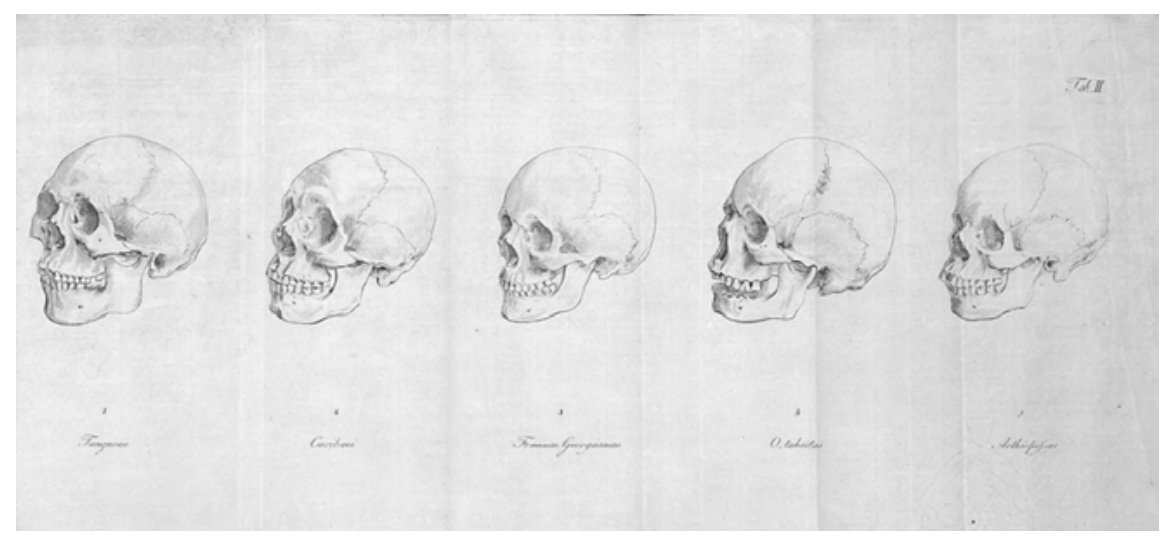

Abb. 6: J.F. Blumenbach: De generis humani varietate nativa (1795): Tab. II.

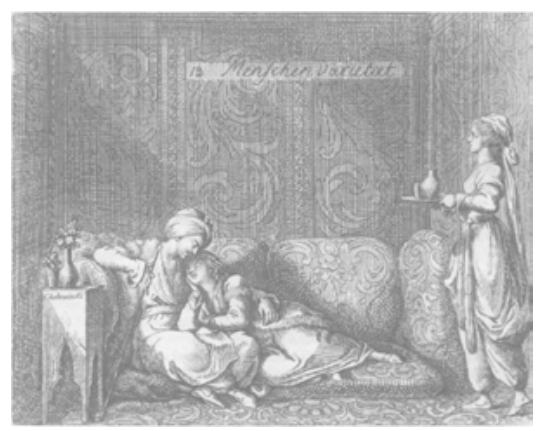

Abb. 7a: J.F. Blumenbach: Beyträge zur Naturgeschichte. Erster Theil (1790): Vignetten der fünf menschlichen Varietäten (von D. Chodowiecki).

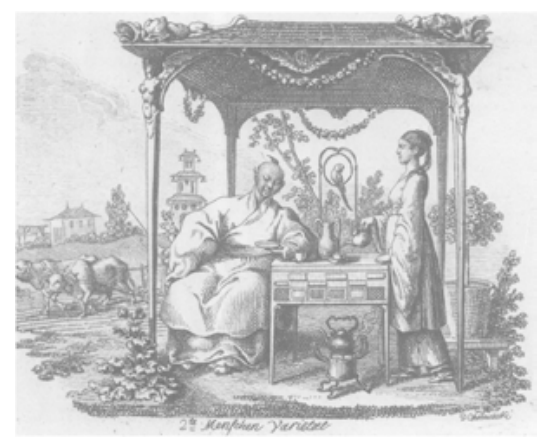

Abb. 7b: J.F. Blumenbach: Beyträge zur Naturgeschichte. Erster Theil (1790): Vignetten der fünf menschlichen Varietäten (von D. Chodowiecki). 


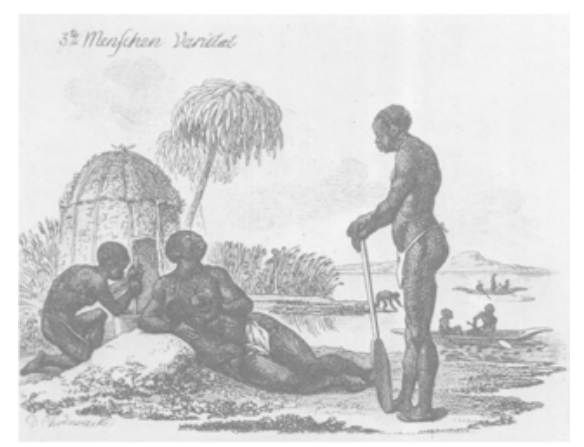

Abb. 7c: J.F. Blumenbach: Beyträge zur Naturgeschichte. Erster Theil (1790): Vignetten der fünf menschlichen Varietäten (von D. Chodowiecki).

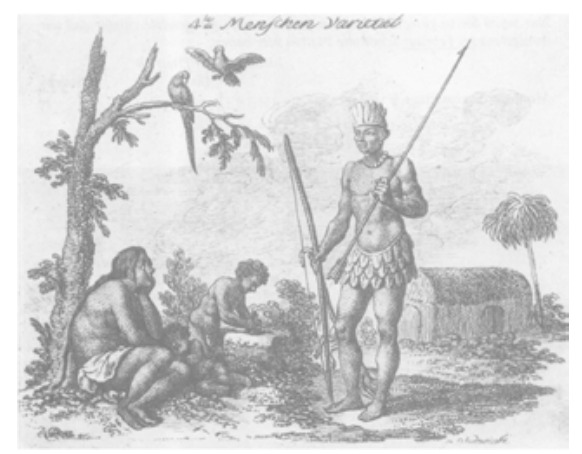

Abb. 7d: J.F. Blumenbach: Beyträge zur Naturgeschichte. Erster Theil (1790): Vignetten der fünf menschlichen Varietäten (von D. Chodowiecki).

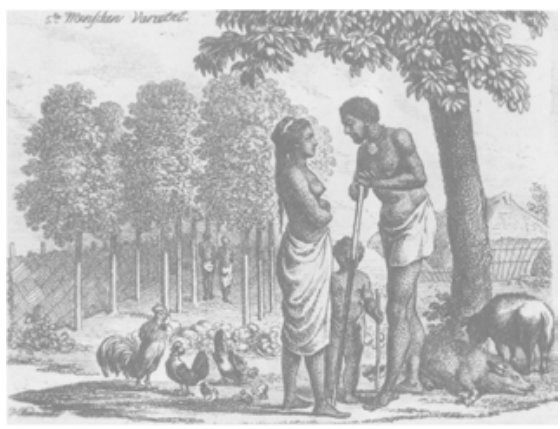

Abb. 7e: J.F. Blumenbach: Beyträge zur Naturgeschichte. Erster Theil (1790): Vignetten der fünf menschlichen Varietäten (von D. Chodowiecki). 


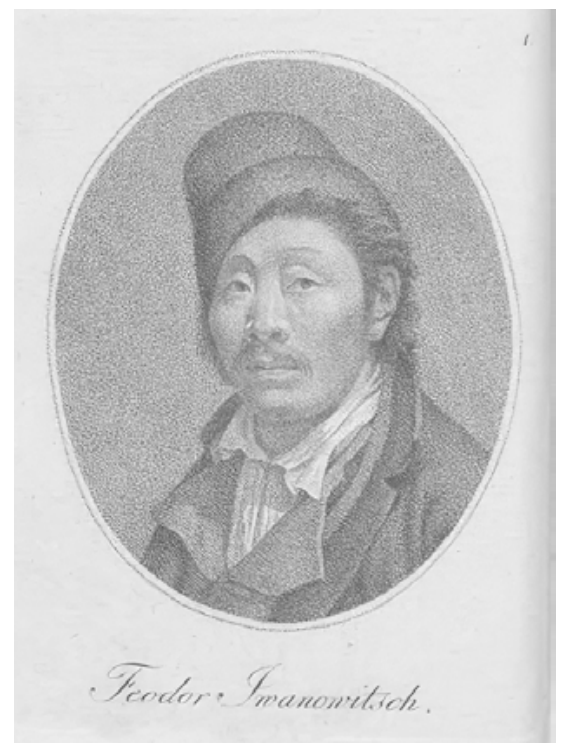

Abb. 8: J.F. Blumenbach: Abbildungen naturhistorischer Gegenstände, 1796-1810-1830. Iwanowitsch. Charakteristischer Musterkopf der Mongolischen Rasse.

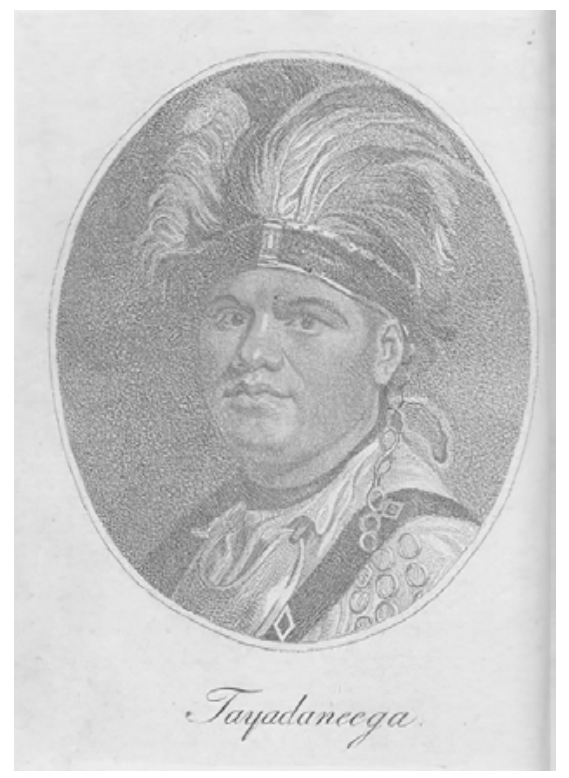

Abb. 9: J.F. Blumenbach, Abbildungen naturhistorischer Gegenstände, 1796-1810-1830. Tayadaneega. Charakteristischer Musterkopf der Americanischen Rasse. 


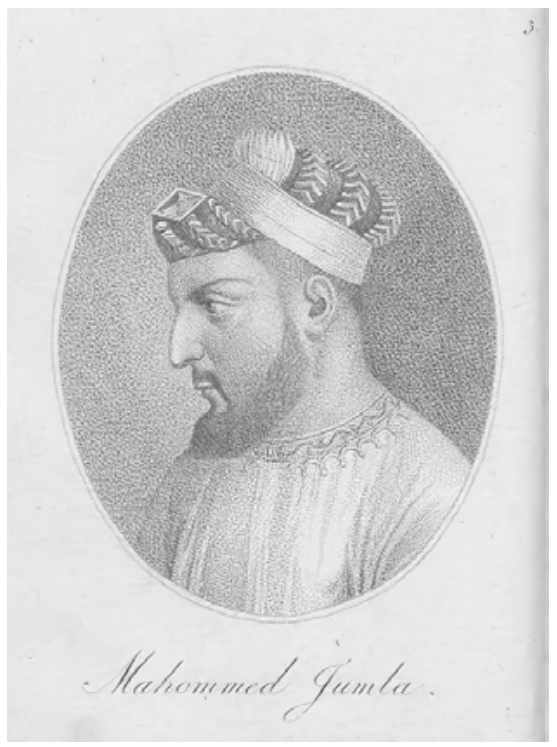

Abb. 10: J.F. Blumenbach, Abbildungen naturhistorischer Gegenstände, 1810-1830². Mahommed Jumla. Charakteristischer Musterkopf der Caucasischen Rasse.

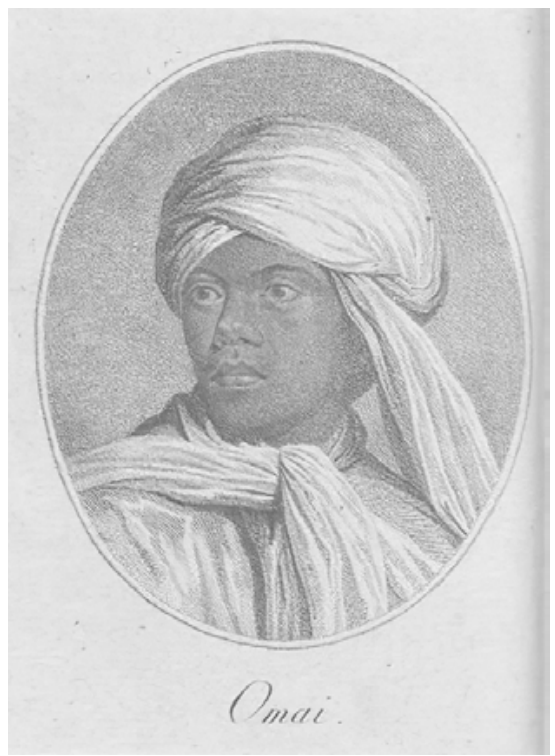

Abb. 11: J. F. Blumenbach, Abbildungen naturhistorischer Gegenstände, 1796-1810-1830. Omai. Charakteristischer Musterkopf der Malayischen Rasse. 


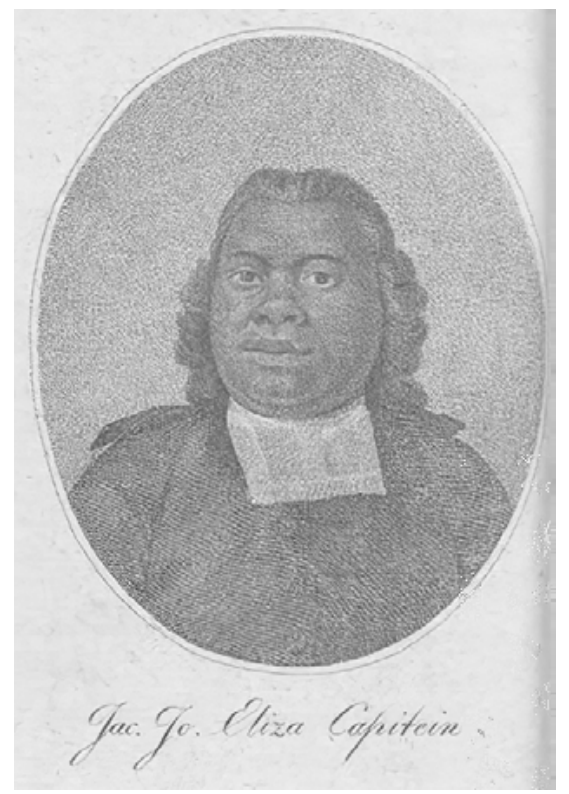

Abb. 12: J. F. Blumenbach, Abbildungen naturhistorischer Gegenstände, 1796-1810-1830. Jaco[bus] Eliza [Elisa] Jo[hannes] Capitein. Charakteristischer Musterkopf der Aethiopischen Rasse.

\section{Literaturverzeichnis}

\section{Primärquellen}

\section{Werke und Briefe von Johann Friedrich Blumenbach}

AG

Blumenbach, Johann Friedrich (1796; 1810; 1830): Abbildungen naturhistorischer Gegenstände. Göttingen: Dieterich.

AT

Blumenbach, Johann Friedrich (1865): The Anthropological Treatises. Übs. von Thomas Bendyshe. London: Longman.

BC 1

Dougherty, Frank William Peter (2006): The Correspondence of Johann Friedrich Blumenbach. Vol. 1: 1773-1782, Letters 1-230. Revised, Augmented and Edited by Norbert Klatt. Göttingen: Norbert Klatt Verlag. BN

Blumenbach, Johann Friedrich (2014): Beyträge zur Naturgeschichte. Hg. von Mario Marino. Reihe: Historia Scientiarum. Hildesheim [et al.]: Olms. 
BN 1790

Blumenbach, Johann Friedrich (1790): Beyträge zur Naturgeschichte. Erster Theil.

Göttingen: Dieterich.

BN 1806

Blumenbach, Johann Friedrich (1806): Beyträge zur Naturgeschichte. Erster Theil (2. Auflage). Göttingen: Dieterich.

CSN

Blumenbach, Johann Friedrich (2018): Contributi alla storia naturale. Hg. von Mario

Marino. Milano: Mimesis.

HN

Blumenbach, Johann Friedrich (1. Auflage 1779, 12. Auflage 1830): Handbuch der Naturgeschichte. Göttingen: Dieterich.

GH 1795

Blumenbach, Johann Friedrich (1795), De generis humani varietate nativa. 3. Auflage.

Göttingen: Vandenhoek \& Ruprecht.

\section{Sekundärquellen}

Baum, Bruce (2006): The Rise and Fall of the Caucasian Race: A Political History of Racial Identity. New York: NYU Press.

Beutel, Alfred (2009): Kirchengeschichte im Zeitalter der Aufklärung. Göttingen: UTB.

Bindman, David (2002): Ape to Apollo. Aesthetics and the Idea of Race in the $18^{\text {th }}$ Century. London: Reaktion Book.

Gehlen, Arnold (1983): Philosophische Anthropologie und Handlungslehre. Frankfurt am Main: Klostermann.

Gehlen, Arnold (2004): Moral und Hypermoral. Eine pluralistische Ethik. Frankfurt am Main: Klostermann.

Gould, Stephen J. (1998): „On Mental and Visual Geometry“. In: Isis 89, 502-503.

Gould, Stephen J. (2002): „The Geometer of Race“. In: Gould, Stephen J.: I have landed. Cambridge: Harvard University Press, 356-366.

Junker, Thomas (1998), „Blumenbach's Racial Geometry“. In: Isis 89, 498-501.

Lawrence, William (1822): Lectures on Physiology, Zoology, and the Natural History of Man. London: Benbow.

Lepenies, Wolf (1976): Das Ende der Naturgeschichte. Frankfurt a. Main: Suhrkamp.

Lévi-Strauss, Claude (2002): Les structures elementaires de la parenté. Berlin: De Gruyter. Marino, Luigi (1975): I maestri della Germania. Göttingen 1770-1820. Torino: Einaudi.

Marino, Mario (2020): „Natural History, Racial Classification and Anthropology in J. F. Blumenbach's Work and Reception“. In: Human Diversity in Context. Hg. von Cinzia Ferrini. Trieste: EUT, 43-73 (open source: http://hdl.handle.net/10077/30261).

Mühlmann, Wilhelm E. (1968): Geschichte der Anthropologie. Wiesbaden: Aula.

Niekerk, Carl (2019): „Zur Narrativisierung der Natur. Johann Friedrich Blumenbachs Beyträge zur Naturgeschichte (1790) zwischen Naturgeschichte und Anthropologie“. In: Rhetorik 38, No. 1, $18-37$.

Schiebinger, Londa (2004): Nature's Body. Gender in the Making of Moderne Science. New Brunswick, NJ: Rutgers University Press. 
Selwyn, Pamela E. (1997): „Daniel Chodowiecki: Der Künstler als Kaufmann“. In: Hinrichs Ernst-Zernack, Klaus (Hg.): Chodowiecki (1726-1801). Kupferstecher, Illustrator, Kaufmann. Tübingen: Niemeyer, 11-21. 